\title{
UK Healthcare Workers' Experiences of Major System Change in Elective Surgery During the COVID-19 Pandemic: Reflections on Rapid Service Adaptation
}

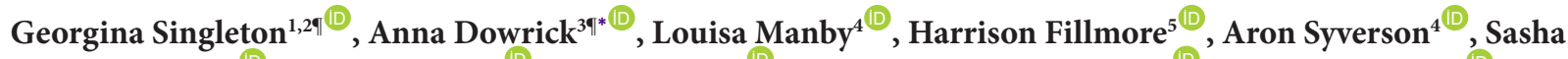 \\ Lewis-Jackson $^{1,2}{ }^{\mathbb{D}}$, Inayah Uddin ${ }^{1,2}{ }^{\mathbb{D}}$, Kirsi Sumray ${ }^{4}$, Elysse Bautista-González ${ }^{4,2}{ }^{\mathbb{D}}$, Ginger Johnson $^{1,2}{ }^{\mathbb{D}}$, \\ Cecilia Vindrola-Padros ${ }^{1,2} \mathbb{D}$
}

\begin{abstract}
Background: The coronavirus disease 2019 (COVID-19) pandemic disrupted the delivery of elective surgery in the United Kingdom. The majority of planned surgery was cancelled or postponed in March 2020 for the duration of the first wave of the pandemic. We investigated the experiences of staff responsible for delivering rapid changes to surgical services during the first wave of the pandemic in the United Kingdom, with the aim of developing lessons for future major systems change (MSC).

Methods: Using a rapid qualitative study design, we conducted 25 interviews with frontline surgical staff during the first wave of the pandemic. Framework analysis was used to organise and interpret findings.

Results: Staff discussed positive and negative experiences of rapid service organisation. Clinician-led decision-making, the flexibility of individual staff and teams, and the opportunity to innovate service design were all seen as positive contributors to success in service adaptation. The negative aspects of rapid change were inconsistent guidance from national government and medical bodies, top-down decisions about when to cancel and restart surgery, the challenges of delivering emergency surgical care safely and the complexity of prioritising surgical cases when services re-started. Conclusion: Success in the rapid reorganisation of elective surgical services can be attributed to the flexibility and adaptability of staff. However, there was an absence of involvement of staff in wider system-level pandemic decisionmaking and competing guidance from national bodies. Involving staff in decisions about the organisation and delivery of MSC is essential for the sustainability of change processes.

Keywords: United Kingdom, Surgery, COVID-19 Pandemic, Healthcare Workers

Copyright: @ 2021 The Author(s); Published by Kerman University of Medical Sciences. This is an open-access article distributed under the terms of the Creative Commons Attribution License (https://creativecommons.org/licenses/ by/4.0), which permits unrestricted use, distribution, and reproduction in any medium, provided the original work is properly cited.

Citation: Singleton G, Dowrick A, Manby L, et al. UK healthcare workers' experiences of major system change in elective surgery during the COVID-19 pandemic: reflections on rapid service adaptation. Int J Health Policy Manag. 2021;x(x):x-x. doi:10.34172/ijhpm.2021.101
\end{abstract}

Article History:

Received: 14 April 2021

Accepted: 7 August 2021

ePublished: 1 September 2021
` Both authors contributed equally to this paper.

\footnotetext{
*Correspondence to:

Anna Dowrick

Email:

anna.dowrick@phc.ox.ac.uk
}

\section{Key Messages}

Implications for policy makers

- Involving staff who are responsible forresponsible for delivering, and are directly impacted by changes to, service delivery in strategic decisionmaking supports the sustainability of major systems change (MSC).

- $\quad$ Enabling and supporting staff to innovate and adapt to service delivery issues is important for rapid implementation.

- National policy-making bodies should provide and regularly update evidence-based guidance to healthcare providers in order to support them to safely and sustainably deliver change during emergencies.

- Guidance from national policy-making bodies should address both the ethical and technical aspects of care delivery during emergencies.

Implications for the public

Coronavirus disease 2019 (COVID-19) led to serious delays in access to elective surgery for patients. This research provides information about how surgical teams in hospitals changed the way they worked in order to minimise risks of spreading COVID-19 while continuing to care for patients. We found that staff felt empowered to make rapid decisions about changes in service provision, but felt excluded from overall decisions about stopping and re-starting surgery. They were anxious about protecting patients from COVID-19, and found it challenging to prioritise who should access surgery first when addressing the backlog of patients. Learning from this research will support ongoing adaptations to the delivery of surgery throughout the pandemic and beyond. 


\section{Background}

The coronavirus disease 2019 (COVID-19) pandemic has placed unprecedented strain on healthcare systems around the world. ${ }^{1}$ In the United Kingdom, rapid reorganisation of care delivery was required due to the National Health Service's (NHS's) inability to cope with patient demand in the context of limited critical care capacity. One of the strategies used to increase capacity was the cancellation and postponement of elective surgery. This enabled a number of other changes. Hospitals were able to use operating theatres and recovery areas as "surge areas" where critical care units could expand, theatre staff were liberated for redeployment and anaesthetic machines were made available for ventilation. Moreover, the flow of patients who did not require urgent care in hospitals was limited, thereby reducing the incidence of intra-hospital infection.

The reorganisation of care delivery at a national scale and within short timeframes provides an opportunity to examine the factors that can act as barriers and enablers of major systems change (MSC) in healthcare systems. MSC involves the planning and implementation of new pathways of care ${ }^{2}$ and shifts in ways of working across multiple healthcare organisations. ${ }^{3}$ Existing research in this field has emphasised that a significant contributor to MSC success is the ability of actors involved in implementing change to make adaptations in line with the opportunities and constraints of their local context. $^{4}$

While there has been substantial description of the overall changes to elective surgery that took place during the pandemic, ${ }^{5-7}$ there is less understanding of healthcare workers' (HCWs) experiences of implementing these service adaptations. Understanding how HCWs navigated and enacted challenging decisions regarding the redesign of elective surgical services during the pandemic will provide lessons for the planning for future MSC, particularly during pandemic conditions.

In this paper, we analyse the experiences of staff involved in adapting the delivery of elective surgery in three UK hospitals during the first wave of the COVID-19 pandemic. In the first part of the paper, we report on the policy context of surgical care during the pandemic. In the second, we provide insights into the positive and negative experiences of reorganisation as perceived by those involved in service delivery during this period of rapid change.

\section{Preparedness for Delivering Elective Surgery During a Pandemic}

Delivering an effective surgical service in the UK NHS was challenging even before the pandemic. A decade of austerity in national spending ${ }^{8}$ had led to the lengthening of waiting times for elective surgery ${ }^{9}$ and a growing incidence of cancellation. ${ }^{10}$ The "UK Influenza Pandemic Preparedness Strategy"11 recognised that, in the case of a severe pandemic, routine elective surgery would be seriously affected. This and other similar policy documents ${ }^{12,13}$ put an emphasis on hospitals making local decisions about how to reorganise services in the event of a pandemic. ${ }^{14}$ A pandemic preparedness simulation run in 2016 identified the need to model the full impact of service closures to inform decision-making as well as the need to develop plans to support the communication of such decisions. ${ }^{15}$

These strategies were put to the test by an unexpectedly severe epidemic of seasonal flu in the winter of 2017/2018. In order to cope with this, all elective surgeries were cancelled, which included all planned, routine non-cancer surgeries from December 2017 to January 2018, leading to a large backlog of surgical procedures. ${ }^{14}$ The advice provided by the NHS stated that each hospital could decide exactly how to manage the reorganisation of services at this time. ${ }^{16}$ In August 2018, this backlog led the NHS to develop an urgent plan to redirect "significantly more" patients to private healthcare providers for their routine procedures. ${ }^{17}$ While this preparation and experience gave some insight into pandemic conditions, further detailed preparations for a pandemic response were not undertaken in UK hospitals.

The COVID-19 Pandemic and UK Elective Surgery Policy Change

As the COVID-19 pandemic progressed, on March 17, 2020, NHS England requested that staff suspend non-urgent elective operations in preparation for the predicted rise in demand for beds, resources and staff availability. ${ }^{18}$ The Royal College of Surgeons (RCS) published guidance on March 20, 2020, which recognised the need for the surgical workforce to adapt during the COVID-19 pandemic. $^{19}$

This approach for managing hospital capacity was similar to that taken in the United States and Italy, where hospitals reduced operating room schedules and removed patients planned for non-essential procedures from operating lists. ${ }^{20,21}$ Other countries, such as South Korea and Singapore, ${ }^{22}$ continued with elective work during the pandemic by adding new control and monitoring measures, such as screening and testing patients before admission and reorganising surgical work into 'hot teams' managing acute surgical admissions and 'cold teams' continuing with elective surgical work.

The RCS recognised the difficult decisions the surgical workforce had to make with regards to prioritising surgical procedures and released further guidance on April 11, 2020 to aid with the decision-making process. ${ }^{5}$ They allocated priority levels to different surgical procedures to help guide the allocation of finite numbers of resources and staff. It was advised that patients needing urgent surgery (such as emergency admissions or cancer treatment), patients who had previously had their procedure delayed, and children should be prioritised. ${ }^{5,18}$

In an attempt to increase labour, resources and facilities to allow the continuation of essential services such as cancer and clinically urgent surgeries, the NHS gained extra capacity from independent, private hospital providers. Private-toNHS hospital conversion for surgical care was operational in the United Kingdom from March 23, 2020. This enabled the NHS to transform independent hospitals into 'COVID-19 light sites. $^{23}$ These sites were specific units or hospitals that provided elective surgical care for non-COVID-19 patients. 


\section{Impact of COVID-19 on Elective Surgery}

Estimates suggest that just over 43300 surgeries were cancelled each week during the 12 -week period in which elective surgical procedures were suspended during the first wave of the pandemic in the United Kingdom. ${ }^{22}$ With the mounting backlog of procedures that this created, the United Kingdom set out to restart elective surgeries on June 18, 2020. ${ }^{24}$ Public Health England published infection and prevention control guidance which outlined that patients undergoing elective surgical procedures should follow a lowrisk COVID-19 pathway. ${ }^{25}$ This meant that patients would need to test and isolate prior to their surgery as well as undergo a clinical assessment of symptoms before their procedure. In addition, emergency rotas were developed and new roles were advertised to reduce the burden of current staff workload and maintain the quality of patient care. ${ }^{26}$

As elective surgeries restarted in the United Kingdom, NHS England set targets that aimed to achieve $80 \%$ of the previous year's surgical activity by the end of September and $90 \%$ by the end of October 2020. ${ }^{6}$ However, even with established COVID-safe pathways and outsourcing to COVID-light sites, a report published on 6 October 2020 by the RCS indicated that surgeons were struggling to reach these targets. ${ }^{27}$ A lack of theatre capacity and sub-optimal levels of staff were major contributing factors. Later in October, several Trusts across the United Kingdom reviewed their position and made the decision to re-suspend non-urgent elective procedures in order to cope with the increasing number of COVID-19 patients during the UK's second wave of the pandemic. ${ }^{28}$ The policy timeline of decisions about suspending and re-starting surgery is summarised in Figure 1.

The sudden onset of the pandemic, the lack of prior specific knowledge about COVID-19 and the lack of robust scientific evidence on the topic made planning surgical services in the first wave of the pandemic extremely difficult. ${ }^{29}$ Early in the pandemic, many of the existing publications around perioperative practice were based on consensus statements and the opinion of expert panels with extrapolated knowledge. ${ }^{30}$ In the United Kingdom, specialist organisations, the Department of Health, Public Health England and the associated Royal Colleges produced national guidance with frequent updates. As the pandemic progressed there was an increase in COVID-19 literature however the level of evidence was often low. ${ }^{31}$ Early reports suggested significant morbidity and mortality associated with surgical patients with concomitant COVID-19 infection. ${ }^{32,33}$ Research later emerged that recognised the associated challenges but suggested that elective surgery could continue safely if appropriate precautions were taken. Kane et al demonstrated that low rates of COVID-19 infection and mortality in the elective surgical population could be achieved by using a targeted care bundle. ${ }^{34}$

Modifications were suggested at every stage of the perioperative pathway in order to protect both staff and

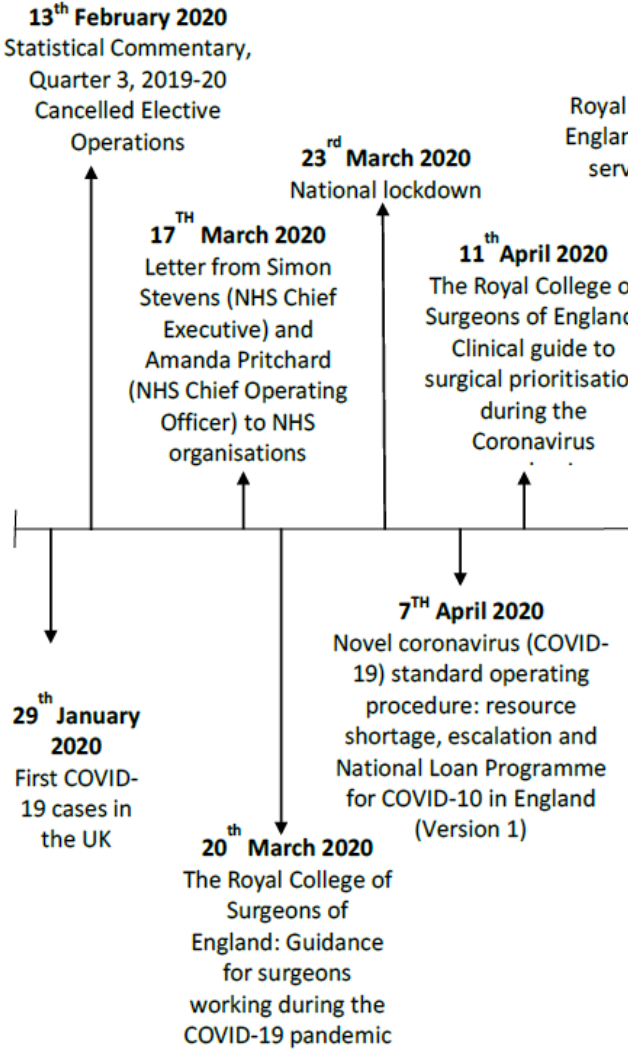

\section{$29^{\text {th }}$ April 2020}

Royal College of Surgeons of England: Recovery of Surgical ervices during and after COVID-19 $8^{\text {th }}$ June 2020

Survey by the Royal College of Surgeons of England: Restart of Elective Surgery after a Pandemic (COVID-19)

$17^{\text {th }}$ September 2020 Novel coronavirus (COVID-19) standard operating procedure: resource shortage, escalation and National Loan Programme for COVID-10 in England (Version 2)

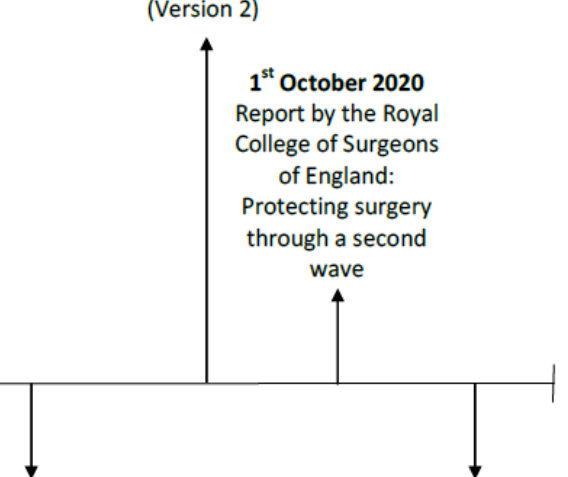

$26^{\text {th }}$ May 2020 Royal College of Surgeons of England: Recovery of Surgical services during and after COVID-19*update to $29^{\text {th }}$ April 2020 guidance

\section{$18^{\text {th }}$ June 2020}

COVID-19: Infection Prevention and Control Guidance (Version 3.2)
$20^{\mathrm{TH}}$ August 2020

COVID-19 Guidance for the Remobilisation of services within health and care setting: infection prevention and control (IPC) recommendations (Version 1.0) *updated version of COVID-19: Infection Prevention and Control Guidance (Version 3.2)
$21^{\text {st }}$ January 2021 COVID-19: Guidance for maintaining services within health care settings Infection prevention and control recommendations (Version 1.1) * updated version of COVID-19: Infection Prevention and Control Guidance (Version 3.2)

Figure 1. Timeline of Events Impacting the Delivery of Elective Surgeries in the United Kingdom. Abbreviations: COVID-19, coronavirus disease 2019; NHS, National Health Service. 
patients from the risks of COVID-19. ${ }^{35}$ Important decisions surrounding surgeries had to be implemented quickly and were often based on day-to-day experiences and reflected the rapidly evolving nature of the situation and the associated evidence. $^{29}$

Reorganisation of Elective Surgery as Major Systems Change We frame the rapid change in the delivery of elective surgery in the United Kingdom during the first wave of the pandemic as an example of MSC. MSC involves introducing specific, targeted changes to the organisation of work within systems which affect a wide number of staff, often framed around the concept of re-designing 'pathways' of care for patients. ${ }^{3}$ Jones et $\mathrm{al}^{36}$ define these processes as:

"Policies, strategies or interventions that aim to transform the way multiple care services are coordinated at the interand intra-organisational level to address a single service area (eg, stroke) or integrated service domain (eg, primary care)."

The MSC field to date has focused on examples of planned system changes, such as centralising service provision, ${ }^{2,37,38}$ integrating different forms of public service, ${ }^{39}$ and introducing new technologies into health systems. ${ }^{40}$ The case study of changes to elective surgery provision during the pandemic enables investigation of the enablers and consequences of unanticipated, rapid change.

While there are a number of characteristics identified as enablers of MSC - unified leadership, locally driven decisions, rapid monitoring and feedback to enable adaptation, and engagement of staff and patients ${ }^{41}$ - it is how these characteristics dynamically manifest in a specific local context that is of particular importance to the success of change processes. ${ }^{42}$ Involvement of those who will be responsible for implementing MSC in decisions about adapting local practices has been identified as crucial to successful implementation, ${ }^{43-45}$ as reorganising service delivery is ultimately achieved by multiple groups of staff collectively adapting their locally established working practices and relationships. ${ }^{36}$ With regard to changes in the provision of surgical services, there has to date been limited investigation of the experiences of staff who implemented these changes. There is growing emphasis on the importance of learning from the adaptations of staff involved in delivering new practices, ${ }^{2,4,46}$ with Turner et al identifying this as particularly important for understanding system responses to the COVID-19 pandemic. ${ }^{1}$ We seek to build on and contribute to this learning through our research.

In this paper, we will contribute to MSC scholarship through an examination of UK HCWs' experiences of reorganising and adapting elective surgical services in response to the COVID-19 pandemic. Adopting a theoretical perspective that MSC is enacted through social practice, ${ }^{47}$ we aimed to develop insights from the perspective of HCWs involved in enacting changes in surgical services during the pandemic. We argue that understanding the enablers of rapid change and reorganisation during the pandemic (from the perspective of those delivering it) contributes broader insights for how policy can guide the effective and safe delivery of elective surgery throughout the duration of the pandemic and beyond.
We focus our analysis on staff's reflections about the positive and negative aspects of rapid service organisation, drawing attention to the perceived barriers and enablers of adapting to pandemic conditions. We provide practical lessons for policymakers and insights about the challenges of implementing MSC in pandemic conditions.

\section{Methods}

This study was part of a larger, ongoing programme of research investigating the perceptions and experiences of HCWs during the COVID-19 pandemic. The wider study utilises a rapid appraisal methodology integrating data from three research streams: telephone interviews with frontline HCWs, UK policy review and media analysis. ${ }^{48}$

For this paper, we drew on in-depth, semi-structured interviews with frontline staff. The aim of the in-depth interviews was to capture the experiences and perceptions of frontline surgical staff in relation to the impact of COVID-19 on the delivery of surgical services. Semi-structured interviews were carried out from March 28, 2020 to June 26, 2020 using a semi-structured topic guide (see Supplementary file 1) which focused on the impact of COVID-19 on elective and emergency surgery delivery, the decision to cancel elective surgeries and the preparedness strategies in place to guide this process as well as the concerns or fears related to the restarting of elective surgeries.

Interviews were conducted via telephone with a purposive sample of 25 frontline staff across three London Trusts which were involved in the wider research programme. ${ }^{49}$ Potential participants were identified by local hospital leads. A sampling framework was developed in order to guide recruitment which included different professional groups, levels of seniority and gender. The final sample included Anaesthetists (8), Emergency Department Doctor (1), Intensive Care Doctor (1), General Physician (1), Surgeons (5), Nurses (4), Healthcare Assistant (1), Services Manager (1) and Allied Health Professionals (3). The surgical specialties included orthopaedics (1), cardiothoracics (1), obstetrics and gynaecology (1) and general surgery (2). Over half of the sample was female, with the majority of participants in middle or senior management positions. Years in practice ranged from less than 1 year to 32 years in service.

These individuals were provided with a participant information sheet and were asked whether they were interested in being contacted by a member of the research team. Where individuals expressed an interest in participating, they were contacted by a researcher who asked if they had any questions about the study. When HCWs agreed to take part in the study, they were asked to sign a consent form. Participants were reminded that their participation was voluntary and that they could withdraw from the study at any time and maintain anonymity. Interviews were audio-recorded and transcribed using an authorised transcription service.

Interview notes were imported into rapid assessment procedures sheets, which were used to synthesise findings on an ongoing basis. ${ }^{49} \mathrm{AD}, \mathrm{GS}, \mathrm{HF}, \mathrm{EBG}, \mathrm{KS}$ and LM collectively read transcripts and developed an analytical coding framework 
based on a preliminary scan of the data using a framework analysis approach. ${ }^{50}$ The coding framework focused on identifying HCW's positive and negative reflections on MSC during the pandemic. These codes were inputted into a Microsoft Excel matrix, with the emerging codes in the columns and interviews entered as individual cases in the rows. The framework was refined during team discussions and all researchers were asked to apply the same framework across their assigned interview transcripts. AD, CV and GJ cross-checked the data during the coding process to ensure consistency across researchers. The resulting themes are summarised in Figure 2.

\section{Results}

Positive Reflections on Rapid Adaptation

\section{Clinician-Led Reorganisation}

Participants in this study attributed the success of the rapid reorganisation of services to changes being clinicianled. While the decision to cancel or delay the majority of elective surgeries 'came from the top' (COV74: Anaesthetic Practitioner), the work of deciding which surgeries to cancel, how to modify care pathways to enable emergency surgeries to continue, and how to re-purpose surgical wards into makeshift intensive care units (ICUs) was done by clinicians. Staff reflected that 'it was a little bit chaotic at times but if you look back, it actually worked quite well' (COV38: Surgeon).

Staff were able to adapt service delivery according to their immediate clinical context. Moreover, usual management-led organisational bureaucracy was to a large extent circumvented. This was also reflected in the liberal allocation of resources, including improved provision of parking and accommodation: 'there is free parking now and multi-storey places to park and also there hotels in place for you to stay' (COV26: Healthcare Assistant). 'Massive changes that normally would have taken us months or years' (COV29: Anaesthetist) were achieved in the space of weeks.

Decisions about how to effectively suspend surgeries and reallocate resources were made at Trust and regional levels, as opposed to nationally. This was facilitated by 'heavy clinical leadership during the pandemic, with teams collaborating together across sites, across hospitals, across specialties' (COV97: Surgeon). This approach enabled consideration of local context, in relation to the surgical infrastructure available and the impact of COVID-19 in a given area at a particular time. For example, some hospitals involved in this study collaborated with other regional hospitals to transform some services into cancer hubs which maintained urgent cancer care.

"Surrounding hospitals would redirect their patients to us, because we were supposed to be COVID free, and we somehow became a cancer hub basically for the north of the city" (COV94: Anaesthetic Practitioner).

A major contributor to rapid adaptation was the ability of staff to collectively draw on their clinical and professional experience to redesign service delivery, collaborating across departments and hospitals to reallocate resources.

\section{Staff and Team Flexibility}

Teams displayed agility in adapting to rapid restructuring, with roles, teams and rotas all regularly changing. Some surgical staff were redeployed to support ICU services while others worked to maintain the provision of emergency care, with regular adjustments to reflect the loss of staff due to illness or requirements for self-isolation.

"We have people who worked Week $A$ and Week $B$ essentially so that if anyone was ill it keeps some of the workforce separate, so we would always have a core workforce who weren't ill" (COV45: Surgeon).

Senior staff in particular were more active in supporting colleagues, with more consultants present overnight to oversee services - 'we used to have no consultant anaesthetist resident overnight, we now have 10 residents in the hospital doing various things' (COV24: Anaesthetist). In some instances, this meant taking responsibility away from junior team members in order to increase efficiency, for example suspending training for junior surgical staff.

"We decided that we would be operating ourselves, between consultants, in order to minimise the risk of complications and in order to speed up the operations" (COV97: Surgeon).

Delivering change quickly was facilitated by improved communication within and between teams - 'there was a chain of communication from, from the top, several times a day, every day' (COV94: Anaesthetist). Rapidly understanding the needs of different teams enabled staff to identify where there
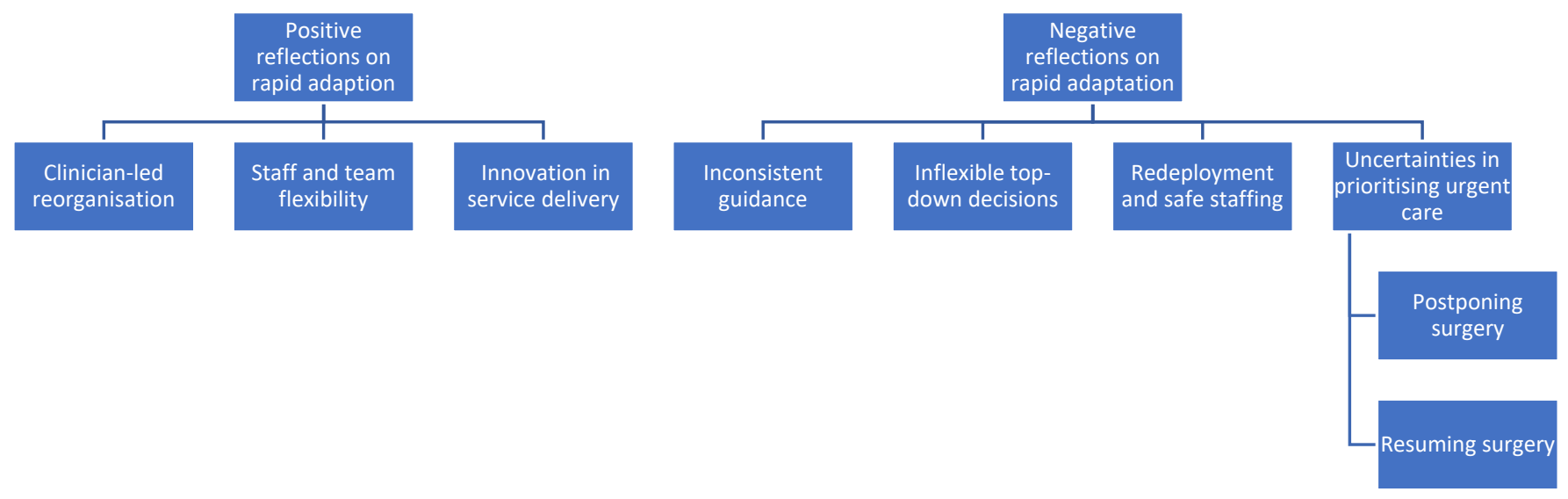

Figure 2. Summary of Themes. 
were gaps in training or equipment and to reallocate resources accordingly.

"There was lots of efforts in grassroots. I was involved in training of airways, in the end I just did it myself because we couldn't wait any longer, for the collective benefit of our colleagues and patients. Management have been very supportive but it is clinicians who have been coming knocking on the door saying we need to prepare and perform these trainings" (COV19: Anaesthetist).

Identifying and responding to local needs regarding the provision of resources, support and training meant that teams could rapidly restructure themselves to adapt to pandemic conditions.

\section{Innovation in Service Delivery}

Despite the challenges of reorganising services, staff felt the way hospitals had been able to react was 'very interesting and exciting' (COV37: Service Manager). A great deal of improvements were thought to stem from the redesign and adaptation of services carried out during the peak of the COVID-19 pandemic.

"Even after the pandemic we definitely want to keep the good things, all the changes we've implemented, because we've seen tangible results in our everyday practice... in terms of time-saving, resource-saving, increasing efficiency, more appropriate managing of resources, managing of staff, all that" (COV37: Services Manager).

This was felt particularly in relation to the flexibility and collaboration between teams and sites, as discussed above, but also with regard to new approaches to caring for patients remotely. Efforts to reduce flow of patients through hospitals meant that staff introduced remote consulting to deliver preoperative care.

"We weren't doing many telephone consultations before but I think we've realised that there are certainly some patients in the future who will be suitable for telephone follow-ups after this which we would have taken a while to realise otherwise" (COV45: Surgeon).

While there were frustrating experiences, such as problems with technology, several HCWs stated that the push into digitalisation during COVID-19 may be beneficial to the wider acceptance of remote care across the perioperative pathway.

"It's been a good opportunity to streamline that, because there's a lot more remotely and in a streamlined process, that we can do it all in one or one or if not, no hospital visits, and do a lot of online, and a lot of it remotely. So hopefully it's something we'll be able to take forward um, once it's all over. Not do it to the same level we're doing it now, but certainly use some of those aspects that keep the health service more efficient" (COV10: Surgeon).

Learning from this experience was also seen as beneficial for preparations for a second peak or a future pandemic. For ongoing uptake of new approaches to be successful, staff suggested that training in innovative practices should continue even when COVID-19 cases are few, informed not only by the UK response but also by insights from other countries.
Negative Reflections on Rapid Adaptation

Inconsistent Guidance

Throughout the COVID-19 pandemic, professional societies and national organisations in the United Kingdom offered guidance about delivering elective procedures. Evidence was also emerging based on surgical experiences from other countries that were affected by COVID-19 earlier than the United Kingdom. Staff felt that they did not have clear advice about how to maintain safety during the surgeries that did proceed.

"We were getting different information every day from different sources, from Europe, within maternity, within the Trust, from PHE [Public Health England], and I think everything felt very, very different...I found that a bit stressful, this conflicting advice and nobody being quite sure what's the right thing to do. Do we break the Trust rules and do what we think is right ourselves?" (COV45: Surgeon). Inconsistent or competing advice from multiple different sources made staff feel in conflict with national bodies.

"Public Health England, were giving out information, so were the WHO, so was our own organisation and then like I said, so were numerous different governing bodies ... and they were all saying something slightly different. So I think that was just extremely difficult to manage and kind of communicate that to, to people" (COV37: Services Manager).

At times they felt they had to fight to protect themselves. For example, some staff described having to negotiate access to personal protective equipment (PPE) for surgical procedures they deemed to be associated with a high risk of COVID-19 transmission but which were not recognised in formal guidance as such. Beyond safety as teams, some participants also felt that they were left to risk-assess their individual risk of COVID-19 exposure.

"I mean that just seems absolutely extraordinary and we put the onus on individuals to say 'I don't think I'm safe to work in COVID areas,' so it was almost like saying, 'it's your problem.' We weren't building it into the system to protect our staff" (COV95: Anaesthetist).

While staff attributed success in service reorganisation to clinician-led decisions about adapting service delivery, they sought more clarity in guidance about delivering surgery safely from Trusts and national bodies to ensure consistency in practice.

"There should be clear guidance from NHS and Government running down to Trust level rather than having to wait for clinicians to change things themselves. Details of certain procedures of course need to be changed for each Trust depending on resources etc but there should be broad lines there from the start" (COV19: Anaesthetist).

Staff argued that local decisions would have been easier within the context of consistent national guidance providing the underlying principles of safe care during the pandemic.

"What has been difficult is the changing guidelines. So this Trust has adhered to the national guidelines but they've changed as the context has changed"(COV29: Anaesthetist). 


\section{Inflexible "Top-Down" Decisions}

The overall decision to postpone non-urgent surgery was considered by the majority of participants to be a 'top-down' decision, with little involvement from frontline clinicians.

"We didn't have much of a say when it came to what was going to get done, whether it was going to get stopped, and when it was going to restart..., we didn't really have that input, or that platform to voice our concerns. It was just 'this is what we're doing, you guys have to do it"' (COV74: Anaesthetic Practitioner).

While the decision to change the model of elective surgery delivery during the pandemic was not contentious among participants in this study, there was ambivalence about the exact way in which services were reorganised. Some staff felt that the decision to stop all elective surgery was 'the right one' (COV85: Surgeon) because of the uncertainty around the risks of surgery at the outset of the pandemic. Equally, other participants reflected that there may have been other approaches for managing risk while continuing to provide surgical services.

"We may not have shut down the whole service in the way that we did. I think we could have had a more strategic plan" (COV95: Anaesthetist).

This uncertainty was motivated by concern about the future consequences. Some feared that the NHS was 'stretching a different problem further down the road' (COV38: Surgeon), with a growing backlog of support needed for patients whose procedures were postponed. Some of the challenge related to the speed at which the decisions were made. Staff noted in particular the delay between awareness of the impact of COVID-19 in Europe and subsequent decisions made about the UK response:

"We could have had a more strategic plan if, as a group we decided to say 'we're going to need to model what's happened in Italy across London but we're going to need to keep elective surgery going, rather than holding off, holding off and then doing a kind of crisis response within a four to six week period" (COV95: Anaesthetist).

HCWs in this study shared a commitment to adapting elective surgery service delivery in light of the pandemic, but displayed considerable doubt in their reflection on both the decision by NHS England and the RCS to cancel all but essential surgical services and its timing.

\section{Redeployment and Safe Staffing}

The cancellation of elective surgical procedures and outpatient clinics led to a reduction in activity in many surgical departments. This reduction in activity combined with a surge in COVID-19 patients meant that many HCWs were redeployed to help with the wider hospital response to the pandemic. The redeployment strategies implemented varied between Trusts, but many HCWs faced redeployment into unfamiliar roles.

"What we've had to do is reconfigure all our staff rotas to support intensive care with its expansion" (COV29: Anaesthetist).

While staff redeployment was considered key to maintaining emergency services and critical care during the pandemic, it was noted to cause anxiety, confusion and stress.

"I think they've found it extremely stressful, some of them have had, you know, they've had anxiety issues, um, and you know, that's because they, I suppose they're going into an area where they know they're going to be dealing with patients that possibly they're not, they don't feel they have the ability to look after" (COV37: Services Manager).

Surgical staff were commonly redeployed to support the busy clinical teams in critical care or on COVID-19 wards but working in this context, often without appropriate skills for their role, was difficult. While formal training was made available, there was not always time to undertake it.

"There's a lot of anxiety amongst the theatre staff and the operating staff and they're all feeling a bit overwhelmed by their experiences in the make-shift ITU that we created. They're all not feeling great" (COV95: Anaesthetist).

Moreover, redeployment was felt to reflect service needs rather than staff skills.

"They redistributed nurses every day. Respiratory trained nurses which would have a better understanding of respiratory problems than a surgical nurse, but those nurses aren't being redeployed to ICU. They were not looking at skill sets, treating each nurse as a number rather than looking at the skills of nurses" (COV22: Ward Sister).

The changing composition of teams, with 'new faces within the department every day' (COV94: Anaesthetist), also made it difficult to establish working practices as a group. Keeping track of rapidly changing processes was also a challenge: 'it was really exhausting just trying to work out what we were doing with different things coming up in different places all the time' (COV45: Anaesthetist).

A concern among all participants was how to manage both surgical activities and COVID-19 care, as it was felt that the hospitals did not have enough staff to 'provide a full set of normal services alongside COVID services' (COV07: Anaesthetist). Staff felt they were being asked to deliver two parallel health systems without sufficient resources: one for COVID-19 and one for non-COVID-19 care.

Though demand for emergency surgery had fallen, with participants describing 'seeing far less people being listed for emergency surgery' (COV07: Anaesthetist) than prior to the pandemic, they argued that it was also critical to maintain an emergency operating service.

Staff described the difficult logistics of delivering emergency surgical care, with concerns relating to case isolation, flow through the hospital, practice in theatres and separation between infected and non-infected areas.

"The key is to separate the hospital into different zones, green zones and potentially infected zones for potentially infected patients to try to mitigate the risk" (COV85: Surgeon).

High levels of staff sickness, which were felt to be 'decimating our service' (COV10: Surgeon) resulted in higher intensity working patterns for those who remained at work. This impacted on the perceived quality and efficiency of surgical teamwork. 
"We used to be a really slick oiled team, we'd have everything ready, but you can see they're all worn out" (COV95: Anaesthetist).

\section{Uncertainties in Prioritising Urgent Care Postponing surgery}

Enacting a reduction in provision of elective surgery involved staff making decisions about which patients' surgeries would be postponed. While the RCS provided guidance on which categories of surgeries should be prioritised, such as surgeries for adults requiring urgent care, staff had to contextualise these decisions within the lives of individual patients, with staff 'very anxious about not exposing people to major surgery and COVID at the same time' (COV95: Anaesthetist). This involved making a judgment about what constituted 'urgent care', balancing the impact of delay on patient outcomes against the demands COVID-19 was placing on the healthcare system.

"My neighbour she was due to have surgery at my hospital next week for a massive prolapse that is painful, that is... it's not life-threatening but it's not life if you cannot walk anywhere because your organs are falling out from your holes, you know. So, she cannot have it and we don't know when she's going to have it, and this is for so many patients" (COV11: Anaesthetist).

Similarly, HCWs were aware of the potential risks of continuing with procedures, for patients and for themselves, particularly during aerosol generating surgeries which were 'highly contagious' (COV74: Anaesthetic Practitioner). For patients who attended in-person appointments, HCWs described higher risk during procedures and higher levels of distress for patients pre- and post-operatively. Some HCWs attributed this to not being allowed someone to accompany them to the hospital and was likely exacerbated by the impersonal nature of being attended to by staff wearing full PPE.

"Post-operatively then they're not allowed any visitors and I think that definitely impacts on their mental health" (COV97: Surgeon).

Deciding whose surgeries would continue involved complex judgment of what discomfort could and should be tolerated by patients, as well as a reflection on the risk of proceeding for both patients and staff.

\section{Resuming Surgery}

HCWs restarted elective surgery following the reduction in COVID-19 cases after the first national lockdown. This represented another major change to service delivery, with staff reporting that it was 'harder to restart elective work than it was to stop and create a new ITU' (COV95: Anaesthetist).

Healthcare staff were widely concerned about the backlog of surgeries and waiting times for patients following the reintroduction of elective and non-urgent surgical procedures. While staff had been able to categorise cases as 'urgent' in the early stages of the pandemic based on immediate threat to life, they faced a significant challenge in deciding where to start among those patients whose surgeries had been postponed: 'we don't really understand how to prioritise' (COV24:
Anaesthetist).

Staff were faced with a number of concurrent challenges. As well as patients whose care had been delayed, other patients needed surgical interventions as a result of their conditions worsening after avoiding hospitals during the pandemic. Decisions about prioritising 'who goes on the waiting list first' (COV45: Surgeon) were taxing. Staff had to weigh up factors that had no clear equivalence, such as whether to prioritise patients based on their risk of further complications, how long they had been waiting, or their current level of pain:

"So, for example, many patients will be on a list for orthopaedic surgery, so all the patients who ... who you know, who have two new hip replacements and knee replacements, things like that, which are debilitating conditions, which aren't necessarily big and life threatening, aren't getting done. But yet they'll still be at home, in their sort of pain or whatever, having to deal with it" (COV10: Surgeon).

Both postponing and restarting surgery involved complex decision-making that left staff unsure about how to 'give people waiting for surgery equitable healthcare' (COV32: Anaesthetist). As well as trying to fairly balance the needs of patients suffering in different ways, staff also had to balance the scale-up of surgery against the potential of a return to high levels of COVID-19 infections. This involved decisions about 'how to do that [reintroduce surgeries] safely and effectively but not get too ahead of the game in case there is a second spike' (COV32: Anaesthetist).

\section{Discussion}

This study aimed to understand the experiences of staff adapting the delivery of UK surgical services during the first wave of the COVID-19 pandemic, interpreting their experiences as a case study of MSC. The success of service adaptation was attributed to clinician-led reorganisation of care into different hubs of localised service delivery which addressed the emergency surgical needs of patients with and without COVID-19. This reflects findings from Turner and colleagues' systematic review of the workforce response to COVID-19, which indicated a move towards 'open leadership' in healthcare systems. ${ }^{1}$ In this respect, the pandemic created conditions which Best et $\mathrm{al}^{41}$ identify as crucial for successful large-scale change: unified leadership around how to respond, locally driven changes, and rapid monitoring and feedback to enable adaptation in light of emerging problems. Reduction in organisational bureaucracy meant that staff were empowered to identify and rapidly implement changes within and between teams, what May et $\mathrm{al}^{4}$ refer to as conditions of greater organisational plasticity.

This case study demonstrates the productive potential of giving staff agency to develop workarounds for problems based on their local knowledge and experience. ${ }^{51,52}$ We join other voices in emphasising the importance of involving those who will deliver change in decisions about how it should be done ${ }^{36,44}$ We build on Myall and colleagues' contention that an overlooked aspect of successful change programmes is how clinical staff interact with and shape multiple aspects of their local context. ${ }^{53}$ In this case, rapid change was possible because staff were given the autonomy to reflect on their local context 
and flexibly adapt existing working practices in a rapidly changing situation. However, we have identified a number of important nuances to this claim.

First, staff were not involved in all decisions about service adaptation. There was minimal consultation of staff involved in service delivery regarding key decisions about cancelling and re-starting elective surgery, despite pre-pandemic policy guidance placing emphasis on hospitals making local decisions about service reorganisation. ${ }^{11-13,15}$ Pressure from COVID-19 was not the same in different Trusts, and staff were frustrated by top-down decisions to cancel non-urgent elective surgery in the absence of critical reflection on alternative service models, as had been done in other countries. ${ }^{22}$ Our findings build upon those of a recent scoping review on the immediate and long-term impact of COVID-19 on the delivery of surgical services, which has argued that contingency plans for continuing with surgical care during the pandemic were missing at a global level and this had a negative impact on patient prognoses, outcomes and experience. ${ }^{54}$

Second, a crucial issue was that the solutions created by staff were temporary, emergency responses. Workarounds, such as those employed by participants in this study, are usually timelimited responses to ongoing structural issues. ${ }^{51}$ While staff lauded their freedom to innovate and the increased investment of organisational resources, they faced a significant challenge in trying to safely and sustainably design and deliver two parallel systems of care for COVID-19 and non-COVID-19 services. Simultaneously delivering both systems within the resources available to them was felt to be untenable. While staff could draw on their clinical knowledge and experience to adapt services, they had limited practical experience regarding the specific requirements of service delivery under pandemic conditions. There was a perceived absence of organisational knowledge and preparedness in this respect.

Finally, staff were placed in a position where they had to choose which surgeries were urgent and which could wait. These were not simple clinical decisions, but complex ethical choices that had to be contextualised within the lives of their patients. ${ }^{55,56}$ The guidance available from national bodies, such as the RCS, ${ }^{5}$ did not sufficiently support staff to engage in these ethical dimensions of care. Struggles to prioritise and cancel surgeries impacted the mental health of HCWs, many who felt overwhelmed and stressed with the backlog of procedures. ${ }^{57}$ Furthermore, conflicting or absent guidance from national bodies about safe delivery of surgery during the pandemic led to ongoing anxiety about infection control for themselves and for patients. ${ }^{58}$

Implications for Policy and Practice

Consistent Guidance on Pandemic Preparedness and Response The inconsistent or contradictory guidance shared with hospital teams created confusion when redesigning service delivery models. Few institutions had organisational knowledge in the form of pandemic preparedness strategies in place. National bodies producing future guidance should ensure that lessons learnt from this and other pandemics are clearly and effectively communicated. This should consider both the ethical and practical dimensions of care-delivery during a pandemic.

\section{Staff Involvement in Decision-Making}

The absence of staff voices in key strategic decisions about the pandemic response had significant consequences for staff experience and patient care. Previous pandemic response guidance advocated for greater autonomy of decision-making at Trust level. Involving staff in ongoing planning should be a priority, enabling staff to contribute their knowledge and experience to decisions about how to sustainably change service provision in response to the affordances and constraints of local context. A range of pragmatic tools and resources for approaching service co-design this are widely available within the improvement and co-production literature, ${ }^{59-61}$ all of which emphasise the importance of clear, ongoing communication between staff delivering changes, management teams and patients.

\section{Ensuring Staff and Patient Safety}

Policy and guidelines now focus on maintaining nonurgent elective operations through subsequent waves of the pandemic. ${ }^{6}$ However, clearing the backlog of surgical procedures is dependent on the ability of staff to safely deliver surgical care. Measures needed to maintain consistent delivery of elective surgical services include safe staffing levels, effective testing of staff and patients and sufficient resources of PPE, drug stocks, theatres and recovery units. ${ }^{62}$

\section{Maintaining Positive Innovations}

The creative response of staff redesigning services led to a number of valuable innovations, such as improved collaboration within and across surgical units and greater uptake of remote pre- and post-operative care. Ensuring that systems and resources are in place that support the sustainable continued uptake of innovation should be prioritised.

\section{Study Limitations}

The findings offered in this paper should be viewed in light the limitations of the study. First, although data were collected during the height of the first wave of the COVID-19 pandemic from a range of staff involved in delivery of surgical services, our respondent pool was mostly comprised of senior staff and was not ethnically diverse. The experiences of more junior staff may have shed light on different issues. Moreover, we only recruited staff from NHS hospitals in London, excluding private hospitals that took on additional NHS surgical work. We did not question staff directly about how they used evidence from the emerging research literature, which may have yielded additional insights.

Despite these limitations we maintain that the overall lessons taken from the case study provide useful insights into sustainable MSC that can be applied throughout the United Kingdom and across other nations with a comparable healthcare system. Their relevance will be amplified by further research exploring how healthcare service delivery has changed in subsequent waves of the pandemic and in other countries. Moreover, it will be important to examine the sustainability of changes over time to see if those that were 
identified as positive remain in place.

\section{Conclusion}

In this paper, we argue that both the successes and challenges in the reorganisation of surgical care during the pandemic are related to the involvement of HCWs in decision-making. Our analysis revealed important contradictions in their experiences. On the one hand, staff were empowered to lead decisions about the practicalities of service re-organisation, enabling creative service adaptations in line with local constraints and opportunities. On the other, there was an absence of involvement of staff in wider system-level pandemic decision-making and unclear guidance about how to continue safely delivering surgery and prioritising who needed it. Limited preparedness and lack of staff involvement ultimately led to short-term gains in terms of infection control but a long-term impact on the delivery of certain services Ensuring sustainable MSC requires effective engagement and involvement of those delivering change.

\section{Acknowledgements}

We would like to acknowledge the support of Dena Javadi, Sam Martin, Sam Vanderslott, Lily-Jay Andrews and Emma Stevens for their support in the early stages of this work.

Ethical issues

The study was reviewed and approved by the Health Research Authority (IRAS: 282069) and the R\&D offices of the hospitals where the study took place.

Competing interests

Authors declare that they have no competing interests.

\section{Authors' contributions}

Conception and design: AD, GS, KS, CVP, GJ. Acquisition of data: KS, HF, EBG, GS, LM, SLJ. Analysis and interpretation of data: KS, HF, EBG, GS, LM, SLJ, AD. Drafting of the manuscript: KS, HF, EBG, GS, LM, SLJ, AD, IU, CVP. Critical revision of the manuscript for important intellectual content: CVP, AD, GS. Supervision: CVP, GJ.

\section{Authors' affiliations}

'Department of Targeted Intervention, University College London, London, UK. ${ }^{2}$ Rapid Research Evaluation and Appraisal Lab (RREAL), University College London, London, UK. ${ }^{3}$ Nuffield Department of Primary Care Health Sciences, University of Oxford, Oxford, UK. ${ }^{4}$ Institute of Epidemiology and Healthcare, University College London, London, UK. ${ }^{5}$ Department of Anthropology, University College London, London, UK.

\section{Supplementary files}

Supplementary file 1. Topic Guide.

\section{References}

1. Turner S, Botero-Tovar N, Herrera MA, et al. Systematic review of experiences and perceptions of key actors and organisations at multiple levels within health systems internationally in responding to COVID-19. Implement Sci. 2021;16(1):50. doi:10.1186/s13012-021-01114-2

2. Fulop NJ, Ramsay Al, Perry C, et al. Explaining outcomes in major system change: a qualitative study of implementing centralised acute stroke services in two large metropolitan regions in England. Implement Sci. 2016;11(1):80. doi:10.1186/s13012-016-0445-z

3. Allen D. From boundary concept to boundary object: the practice and politics of care pathway development. Soc Sci Med. 2009;69(3):354-361. doi:10.1016/j.socscimed.2009.05.002

4. May CR, Johnson M, Finch T. Implementation, context and complexity. Implement Sci. 2016;11(1):141. doi:10.1186/s13012-016-0506-3

5. Royal College of Surgeons of England. Clinical Guide to Surgical Prioritisation during the Coronavirus Pandemic - Royal College of
Surgeons. https://www.rcseng.ac.uk/coronavirus/surgical-prioritisationguidance/. Accessed March 15, 2021. Published 2020.

6. Royal College of Surgeons of England. Recovery of Surgical Services during and after COVID-19 - Royal College of Surgeons. https://www. rcseng.ac.uk/coronavirus/recovery-of-surgical-services/. Accessed March 15, 2021. Published May 26, 2020.

7. Department of Health. Resource Shortage, Escalation and National Loan Programme for COVID-19 in England. https://www.england.nhs. uk/coronavirus/publication/resource-shortage-escalation-and-nationalloan-programme-for-covid-19-in-england/. Accessed March 15, 2021. Published April 7, 2020.

8. Kerasidou A, Kingori P. Austerity measures and the transforming role of A\&E professionals in a weakening welfare system. PLoS One. 2019; 14(2):e0212314. doi:10.1371/journal.pone.0212314

9. NHS England. Consultant-Led Referral to Treatment Waiting Times Data 2019-2020. https://www.england.nhs.uk/statistics/statistical-work-areas/ rtt-waiting-times/rtt-data-2019-20/. Accessed March 15, 2021. Published 2020.

10. NHS England. Statistics on Cancelled Elective Operations. https:// www.england.nhs.uk/statistics/statistical-work-areas/cancelled-electiveoperations/. Accessed March 15, 2021. Published 2020.

11. Department of Health. UK Influenza Pandemic Preparedness Strategy 2011. https://assets.publishing.service.gov.uk/government/uploads/ system/uploads/attachment_data/file/213717/dh_131040.pdf. Accessed March 15, 2021. Published 2011.

12. NHS England. Operating Framework for Managing the Response to Pandemic Influenza. https://www.england.nhs.uk/publication/operatingframework-for-managing-the-response-to-pandemic-influenza/. Accessed March 15, 2021. Published 2017.

13. Kent Resilience Forum. Pandemic Influenza Contingency Plan. Kent Resilience Forum; 2009.

14. Herrod PJJ, Adiamah A, Boyd-Carson H, et al. Winter cancellations of elective surgical procedures in the UK: a questionnaire survey of patients on the economic and psychological impact. BMJ Open. 2019; 9(9):e028753. doi:10.1136/bmjopen-2018-028753

15. Public Health England. Exercise Cygnus Report: Tier One Command Post Exercise Pandemic Influenza. https://assets.publishing.service.gov. uk/government/uploads/system/uploads/attachment_data/file/927770/ exercise-cygnus-report.pdf. Accessed March 15, 2021. Published 2016.

16. North Tees and Hartlepool NHS Foundation Trust. Outpatient Appointments and Elective Surgery Cancellations; 2018. https://www. nth.nhs.uk/news/outpatient-appointments-elective-surgery-cancellationsstatement/. Accessed April 9, 2021.

17. Illman J. More patients could be sent private as waiting list spirals. Health Service Journal website. https://www.hsj.co.uk/quality-andperformance/exclusive-more-patients-could-be-sent-private-as-waitinglist-spirals/7023242.article. Accessed April 9, 2021. Published August 30, 2018.

18. Simon Stevens, Amanda Pritchard. Next Steps on NHS Response to COVID-19. https://www.england.nhs.uk/coronavirus/wp-content/uploads/ sites/52/2020/03/20200317-NHS-COVID-letter-FINAL.pdf. Accessed March 15, 2021. Published 2020.

19. Royal College of Surgeons of England. Guidance for Surgeons Working during the COVID-19 Pandemic. https://www.rcseng.ac.uk/coronavirus/ joint-guidance-for-surgeons-v1/. Accessed March 15, 2021. Published March 20, 2020.

20. Lancaster EM, Sosa JA, Sammann A, et al. Rapid response of an academic surgical department to the COVID-19 pandemic: implications for patients, surgeons, and the community. J Am Coll Surg. 2020;230(6):1064-1073. doi:10.1016/j.jamcollsurg.2020.04.007

21. Spinelli A, Pellino G. COVID-19 pandemic: perspectives on an unfolding crisis. Br J Surg. 2020;107(7):785-787. doi:10.1002/bjs.11627

22. Nepogodiev D, Omar OM, Glasbey JC, et al. Elective surgery cancellations due to the COVID-19 pandemic: global predictive modelling to inform surgical recovery plans. Br J Surg. 2020;107(11):1440-1449. doi:10.1002/ bjs. 11746

23. McCabe R, Schmit N, Christen P, et al. Adapting hospital capacity to meet changing demands during the COVID-19 pandemic. BMC Med. 2020; 18(1):329. doi:10.1186/s12916-020-01781-w

24. Macdonald N, Clements C, Sobti A, Rossiter D, Unnithan A, Bosanquet $\mathrm{N}$. Tackling the elective case backlog generated by COVID-19: the scale of the problem and solutions. J Public Health (Oxf). 2020;42(4):712-716. 
doi:10.1093/pubmed/fdaa155

25. Public Health England. COVID-19: Guidance for Maintaining Services within Health and Care Settings. https://assets.publishing.service.gov. uk/government/uploads/system/uploads/attachment_data/file/954690/ Infection_Prevention_and_Control_Guidance_January_2021.pdf. Accessed March 15, 2021. Published 2020.

26. Chapman R. COVID-19: impacting the acute surgical assessment. $\mathrm{Br} \mathrm{J}$ Surg. 2020;107(11):e515. doi:10.1002/bjs.11984

27. Royal College of Surgeons. Protecting Surgery through a Second Wave. https://www.rcseng.ac.uk/coronavirus/protecting-surgery-through-asecond-wave/. Accessed March 15, 2021. Published October 1, 2020.

28. ABHI. NHS Restart: Briefing Document. https://www.abhi.org.uk/ media/2683/the-restart-briefing-document.pdf. Accessed March 15, 2021. Published 2020.

29. Bresadola V, Biddau C, Puggioni A, et al. General surgery and COVID-19: review of practical recommendations in the first pandemic phase. Surg Today. 2020;50(10):1159-1167. doi:10.1007/s00595-020-02086-4

30. Shanthanna H, Uppal V. Surgery during the COVID-19 pandemic. Lancet. 2020;396(10261):e74.doi:10.1016/s0140-6736(20)32279-0

31. Wielogórska NL, Ekwobi CC. COVID-19: what are the challenges for NHS surgery? Curr Probl Surg. 2020;57(9):100856. doi:10.1016/j. cpsurg.2020.100856

32. Lei S, Jiang F, Su W, et al. Clinical characteristics and outcomes of patients undergoing surgeries during the incubation period of COVID-19 infection. EClinicalMedicine.2020;21:100331.doi:10.1016/j.eclinm.2020.100331

33. Nepogodiev D, Bhangu A, Glasbey JC, et al. Mortality and pulmonary complications in patients undergoing surgery with perioperative SARSCoV-2 infection: an international cohort study. Lancet. 2020;396(10243):2738. doi:10.1016/s0140-6736(20)31182-x

34. Kane AD, Paterson J, Pokhrel S, et al. Peri-operative COVID-19 infection in urgent elective surgery during a pandemic surge period: a retrospective observational cohort study. Anaesthesia. 2020;75(12):1596-1604. doi:10.1111/anae.15281

35. Al-Jabir A, Kerwan A, Nicola M, et al. Impact of the coronavirus (COVID-19) pandemic on surgical practice - part 1. Int J Surg. 2020;79:168-179. doi:10.1016/j.ijsu.2020.05.022

36. Jones L, Fraser A, Stewart E. Exploring the neglected and hidden dimensions of large-scale healthcare change. Sociol Health IIIn. 2019; 41(7):1221-1235. doi:10.1111/1467-9566.12923

37. Fulop N, Walters R, Perri, Spurgeon P. Implementing changes to hospital services: factors influencing the process and 'results' of reconfiguration. Health Policy. 2012;104(2):128-135. doi:10.1016/j.healthpol.2011.05.015

38. Vallejo-Torres L, Melnychuk M, Vindrola-Padros C, et al. Discrete-choice experiment to analyse preferences for centralizing specialist cancer surgery services. Br J Surg. 2018;105(5):587-596. doi:10.1002/bjs.10761

39. Maniatopoulos G, Hunter DJ, Erskine J, Hudson B. Large-scale health system transformation in the United Kingdom. J Health Organ Manag. 2020;34(3):325-344. doi:10.1108/jhom-05-2019-0144

40. Greenhalgh T, Russell J, Ashcroft RE, Parsons W. Why national eHealth programs need dead philosophers: wittgensteinian reflections on policymakers' reluctance to learn from history. Milbank Q. 2011;89(4):533563. doi:10.1111/j.1468-0009.2011.00642.x

41. Best A, Greenhalgh T, Lewis S, Saul JE, Carroll S, Bitz J. Large-system transformation in health care: a realist review. Milbank Q. 2012;90(3):421456. doi:10.1111/j.1468-0009.2012.00670.x

42. Harvey G, Kitson A. Translating evidence into healthcare policy and practice: Single versus multi-faceted implementation strategies - is there a simple answer to a complex question? Int J Health Policy Manag. 2015; 4(3):123-126. doi:10.15171/ijhpm.2015.54

43. Martin GP, Carter P, Dent M. Major health service transformation and the public voice: conflict, challenge or complicity? J Health Serv Res Policy. 2018;23(1):28-35. doi:10.1177/1355819617728530

44. McKevitt C, Ramsay AIG, Perry C, et al. Patient, carer and public involvement in major system change in acute stroke services: the construction of value. Health Expect. 2018;21(3):685-692. doi:10.1111/ hex. 12668

45. Willson A, Davies A. Rhetoric or reform? changing health and social care in Wales. Int J Health Policy Manag. 2021;10(6):295-298. doi:10.34172/ ijhpm.2020.53

46. Bate P, Robert G, Fulop N, Øvretveit J, Dixon-Woods M. Perspectives on Context: A Selection of Essays Considering the Role of Context in Successful Quality Improvement. https://www.health.org.uk/sites/health/ files/PerspectivesOnContext_fullversion.pdf. Accessed July 15, 2018. Published 2014.

47. May C, Finch T. Implementing, embedding, and integrating practices: an outline of normalization process theory. Sociology. 2009;43(3):535-554. doi: $10.1177 / 0038038509103208$

48. Vindrola-Padros C, Andrews L, Dowrick A, et al. Perceptions and experiences of healthcare workers during the COVID-19 pandemic in the UK. BMJ Open. 2020;10(11):e040503. doi:10.1136/bmjopen-2020-040503

49. Vindrola-Padros $C$, Chisnall $G$, Cooper S, et al. Carrying out rapid qualitative research during a pandemic: emerging lessons from COVID-19. Qual Health Res. 2020;30(14):2192-2204. doi:10.1177/1049732320951526

50. Gale NK, Heath G, Cameron E, Rashid S, Redwood S. Using the framework method for the analysis of qualitative data in multi-disciplinary health research. BMC Med Res Methodol. 2013;13:117. doi:10.1186/1471 2288-13-117

51. Alter S. Theory of workarounds-draft manuscript. Commun Assoc Inf Syst. 2013;34(55):1-30.

52. Zhou X, Ackerman MS, Zheng K. CPOE Workarounds, Boundary Objects, and Assemblages. https://socialworldsresearch.org/sites/default/files/ chi11-zhou-CPOE.pdf. Accessed March 20, 2018.

53. Myall M, May C, Richardson A, et al. Creating pre-conditions for change in clinical practice: the influence of interactions between multiple contexts and human agency. J Health Organ Manag. 2020;35(9):1-17. doi:10.1108/ jhom-06-2020-0240

54. Søreide K, Hallet J, Matthews JB, et al. Immediate and long-term impact of the COVID-19 pandemic on delivery of surgical services. $\mathrm{Br} J$ Surg. 2020;107(10):1250-1261. doi:10.1002/bjs.11670

55. Newdick C, Sheehan M, Dunn M. Tragic choices in intensive care during the COVID-19 pandemic: on fairness, consistency and community. J Med Ethics. 2020;46(10):646-651. doi:10.1136/medethics-2020-106487

56. Huxtable R. COVID-19: where is the national ethical guidance? BMC Med Ethics. 2020;21(1):32. doi:10.1186/s12910-020-00478-2

57. Vera San Juan N, Aceituno D, Djellouli N, et al. Mental health and wellbeing of healthcare workers during the COVID-19 pandemic in the UK: contrasting guidelines with experiences in practice. BJPsych Open. 2020; 7(1):e15. doi:10.1192/bjo.2020.148

58. Hoernke K, Djellouli N, Andrews L, et al. Frontline healthcare workers' experiences with personal protective equipment during the COVID-19 pandemic in the UK: a rapid qualitative appraisal. BMJ Open. 2021; 11(1):e046199. doi:10.1136/bmjopen-2020-046199

59. Robert G, Williams O, Lindenfalk B, et al. Applying Elinor Ostrom's Design Principles to Guide Co-Design in Health(care) Improvement: A Case Study with Citizens Returning to the Community from Jail in Los Angeles County. Int J Integr Care. 2021;21(1):7. doi:10.5334/ijic.5569

60. Batalden $M$, Batalden $P$, Margolis $P$, et al. Coproduction of healthcare service. BMJ Qual Saf. 2016;25(7):509-517. doi:10.1136/ bmjqs-2015-004315

61. Pannick S, Sevdalis N, Athanasiou T. Beyond clinical engagement: a pragmatic model for quality improvement interventions, aligning clinical and managerial priorities. BMJ Qual Saf. 2016;25(9):716-725 doi:10.1136/bmjqs-2015-004453

62. Royal College of Surgeons. Elective Surgery during COVID-19. https:// www.rcseng.ac.uk/news-and-events/news/archive/survey-resultselective-surgery-under-covid/. Accessed April 9, 2021. Published June 26, 2020. 